\title{
Atomic diffusion and element mixing in pulsating stars
}

\author{
Georges Alecian \\ LUTH (Observatoire de Paris - CNRS), Observatoire de Meudon, F-92190 Meudon, France, \\ email: georges.alecian@obspm.fr
}

\begin{abstract}
Stellar plasmas are multicomponent anisotropic gases. Each component (chemical element) of these gases experiences specific forces related to its properties, which leads each element to diffuse with respect to the others. There is no reason why a stellar plasma should remain homogeneous except if mixing motions enforce homogeneity. Because atomic diffusion is a very slow process, the element separation only occurs in places where mixing motions are weak enough not to erase the effect of the ineluctable tendency of chemical elements to migrate. In this talk, I will present how atomic diffusion and mixing processes compete in stars (interiors as well as atmospheres), and I will show various cases where atomic diffusion is believed to have noticeable effects. This concerns several types of stars throughout the H-R diagram, including pulsating ones.
\end{abstract}

Keywords. atomic processes, diffusion, turbulence, stars: mass loss, stars: abundances

\section{Introduction}

In multicomponent gases, diffusion is usually considered as a physical process which tends to homogenize mixtures by smoothing concentration gradients. Therefore, it is familiar to consider the second spatial derivative of the particle number density $n_{k}$ in the continuity equation for the species $k$ (in the plane-parallel case):

$$
\partial_{t} n_{k}+\ldots+D_{k} \cdot \partial_{z}^{2} n_{k}=0
$$

where $D_{k}$ is the diffusion coefficient for $k$. No advective term appears in this expression. However, in modeling atomic diffusion in stars, one often speaks about diffusion velocity of elements, which may sound odd to some physicists. Actually, following Chapman \& Cowling (1970) one can define in the framework of the kinetic theory of gases the relative velocities $V_{k t}$ of the species $k$ with respect to each of the other components $t$ of the mixture (see Alecian \& Michaud 2005) by:

$$
\sum_{t} \frac{p_{k} p_{t}}{p D_{k t}} V_{k t}=A_{k}
$$

where $p_{k}, p_{t}$ and $p$ are the partial and total pressures respectively, $D_{k t}$ is the diffusion coefficient of $k$ with respect to $t$ (related to the collision rate between these two species), and $A_{k}$ a term gathering all the forces acting on particle $k$ (gravity, radiation force, etc...). The relative velocities $V_{k t}$ have a statistical meaning in the sense that they are obtained from the average deviation of each species from their thermal velocity. In a star, if one considers that metals are trace elements in a gas dominated by protons and electrons, one can solve for the diffusion velocities $V_{D_{k}}$ of each metal with respect to the stellar plasma. This velocity is then an average velocity of particles belonging to the population of type $k$, and it may be introduced in the form of an additional advective term due to atomic diffusion in Eq. 1.1. However, no fluid motion can be related to this velocity; it is 
the velocity of the displacement of the population of $k$ inside the medium, which could be at rest or moving. Speaking about diffusion velocities of metals in the context of stellar modeling, one refers generally to this picture. Notice that, in advanced stellar modeling, metals (including $\mathrm{He}$ ) are generally not considered as trace elements, which complicates significantly the equations describing the process.

In Sect. 2, I will discuss in more detail the diffusion velocity, and I will show how one can formulate the competition of atomic diffusion and mixing processes. In Sect. 3, I will present the case of AmFm stars where atomic diffusion is known to act in internal radiative zones. Sect. 4 will be devoted to hotter main-sequence stars.

\section{Mixing and mass loss vs. atomic diffusion}

We consider here the simple case of the diffusion of a trace element in ionization state $i$ (charge $Z_{i}$ ), with respect to protons in a ternary mixture (ions-protons-electrons). In a formulation inspired from Chapman \& Cowling (1970), the diffusion velocity may be written as:

$$
V_{i p} \approx \underbrace{-\left(D_{i p}+D_{\text {turb }}\right) \partial_{r} \ln \frac{n_{i}}{n_{p}}}_{(1)}+D_{i p}[\underbrace{A_{i} \frac{m_{p}}{k T}\left(g_{i}^{\mathrm{rad}}-g\right)}_{(2)}-\underbrace{\left(\frac{Z_{i}+1}{2}\right) \frac{m_{p}}{k T} g}_{(3)}+\ldots],
$$

where $m_{p}$ is the proton mass, $A_{i}$ the atomic mass, $k$ the Boltzmann constant, and $T$ the temperature.

One can highlight three main terms as indicated in the expression above (the term due to thermal diffusion is not shown here). The first term (1) may be called the pure diffusion term. This term corresponds to the usual smoothing process mentioned in commenting Eq. 1.1. In this first term, a turbulent diffusion coefficient $\left(D_{\text {turb }}\right)$ has been added to the microscopic diffusion coefficient by Schatzman (1969) to account for turbulent mixing. The second term (2) corresponds to the competition of gravity against the radiative force, as introduced by Michaud (1970). The third term (3) was introduced by Aller \& Chapman (1960) and corresponds to the effect of the electric field due to diffusion of electrons. The diffusion velocity of an element $k$ may be estimated by computing a weighted average of the ion velocities.

The continuity equation for the $k$ particle number density may be written as:

$$
\partial_{t} n_{k}+\nabla\left[\mathrm{n}_{\mathrm{k}} \cdot\left(\mathbf{V}_{\mathrm{D}_{\mathrm{k}}}+\mathbf{V}_{\mathrm{M}}\right)\right]=0,
$$

where $\mathbf{V}_{\mathrm{M}}$ is a macroscopic velocity of the plasma (e.g., stellar wind, large scale circulation). So, both processes (atomic diffusion and mixing) are gathered in the expression of the diffusion velocity. Outside radiative zones, mixing processes are so strong and $D_{\text {turb }}$ so large in term (1) of Eq. 2.1, that all the other terms are negligible. In that case, one recovers the continuity equation in the form of Eq. 1.1 with just the smoothing term (with $D_{k}=D_{\text {turb }}$ ). On the contrary, in radiative zones, $D_{\text {turb }}=0$, and the smoothing term (pure diffusion) can have some efficiency only after strong abundance gradients have been built up by atomic diffusion dominated by the competition between gravity and radiative acceleration in term (2).

The wind velocity $\mathbf{V}_{\mathrm{M}}$ may be modeled as a radial flow of matter. It may be simply obtained assuming the conservation of a constant mass flux in spherical geometry:

$$
V_{\mathrm{M}}(r)=\dot{M}_{\mathrm{star}}\left(4 \pi \rho r^{2}\right)^{-1}
$$




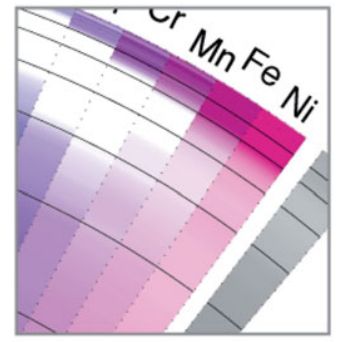

a

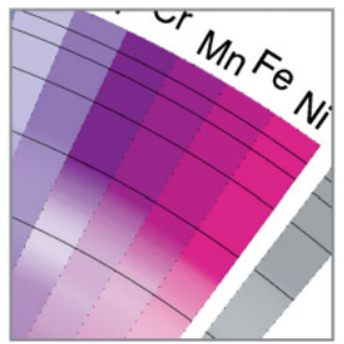

$\mathrm{b}$

Figure 1. This figure is a magnified part of Fig. 5 in Michaud et al. (2011) (the reader is encouraged to see the original legend in their paper for color code, scales, and model details). It shows the concentration of some iron-peak elements in the upper layers of a main-sequence A star with the same fundamental parameters as Sirius A (see Landstreet 2011). In the left panel (the case assuming mass loss), metals are concentrated in high layers compared to the right panel (without mass loss) where overabundances extend deeper.

where $\dot{M}_{\text {star }}$ is the mass-loss rate and $\rho$ the mass density at radius $r$. The stellar mass loss has a special status because it is not a mixing process, but it contributes to homogenize the medium. Indeed, if $\mathbf{V}_{\mathrm{M}} \gg \mathbf{V}_{\mathrm{D}_{k}}$, the local mixture is replaced by fresh homogeneous mixture (brought by the mass-loss flux from deep layers) faster than atomic diffusion can build up abundance stratifications.

\section{The case of AmFm stars}

The case of AmFm stars may illustrate both the role of turbulent mixing and mass loss in modeling the stellar interiors. AmFm stars constitute the cooler group of chemically peculiar stars of the main sequence (in the $T_{\text {eff }}$ range $7000-10000 \mathrm{~K}$ ). To explain AmFm stars, one usually considers a first phase when, because of the low rotation of these stars, atomic diffusion of helium (gravitational settling) leads to a decrease of the depth of the outer convection zone. A second phase, when heavier elements diffuse in a radiative zone, follows. After the first phase, this radiative zone has a higher upper boundary where atomic diffusion is more efficient (see Charbonneau \& Michaud 1988). Alecian (1996) has proposed that, to explain the systematic underabundances of Ca and Sc in AmFm stars, it is necessary to assume a small mass loss in addition to atomic diffusion. Some years later, Richer et al. (2000) who developed the Montreal evolution code (with no mass loss), had shown that during the second phase, the iron accumulation may trigger a new convection zone that makes deeper the upper boundary of the radiative zone. Such a new mixing zone in the higher part of the radiative zone appears to be still compatible with most (but not all, see below the case of Sc) of the abundance anomalies observed in AmFm stars.

A new version of the Montreal evolution code (Vick et al. 2010) has been applied by Michaud et al. (2011) to the case of Sirius A, which is a hot Am star (see Landstreet 2011). Figure 1 shows two computations realized by Michaud et al. (2011). The left panel shows the result assuming mass loss, while the right panel corresponds to the case with no mass loss. Both models give surface abundance pattern close to the observed one, but quite different abundance stratifications in the interior. To know which one of these two models is the closest to real stars, Michaud et al. (2011) suggest that asteroseismology could help to distinguish between mass loss and turbulence as dominant process for abundance stratifications in AmFm stars. On the other hand, Alecian et al. (2013) discussed the case of scandium in AmFm stars and have instead confirmed the result of Alecian (1996) 
and Leblanc \& Alecian (2008), who find that underabundances of Sc in AmFm stars requires that diffusion of Sc occur just below the superficial convection of $\mathrm{H}$ (without a convection zone due to iron), with a small mass loss. So, the presence of mass loss in AmFm stars (and for the hotter HgMn stars) remains a subject of debate which could benefit from asteroseismology.

Another interesting aspect concerning AmFm stars is the fact that they share the same region of the $\mathrm{H}-\mathrm{R}$ diagram as $\delta$ Scuti stars. The classical model has considered that there was a dichotomy of the two groups: slowly rotating stars become AmFm because of helium settling, and fast rotators become $\delta$ Scuti stars because helium remains in the outer layers and can trigger the $\kappa$ mechanism. One knows that the situation is not so simple. Apart from the coexistence with, for instance, $\lambda$ Boo stars, and $\delta$ Del stars in the same region of the H-R diagram, some Am stars pulsating like $\delta$ Scuti stars have been observed at the red edge of the instability strip. However, one can still consider that the main features of the classical model remain valid, and that evolution models need to be refined to understand intermediate cases, or stars transiting from one subgroup to the other.

\section{Hotter stars}

\subsection{The case of chemically peculiar stars}

Considering hotter main-sequence stars leads us first to look at the ApBp chemically peculiar stars (we will not consider here He-weak or He-rich type stars). This group is composed by three subgroups (roAp, magnetic ApBp, $\mathrm{HgMn}$ ), which share a main characteristic: atomic diffusion occurs in their atmospheres. Because atomic diffusion is more efficient when particle density is weak, abundance anomalies are much stronger in these stars than in AmFm stars.

As discussed by Michaud (1970), several properties of these stars allow one to assume that either there are no mixing processes in their atmospheres, or that these processes are not strong enough to compete with atomic diffusion (slow rotation, not enough $\mathrm{He}$ and too hot atmospheres to have convection, very strong magnetic fields, etc.). During several decades, modeling of atomic diffusion in ApBp stars relied on this assumption of stability. Even if this assumption was justified by several serious arguments, there were no observational measurements to confirm it. This is no longer the case, since many recent spectroscopic observations show evidence of abundance stratifications in the atmospheres of ApBp stars (Ryabchikova et al. 2002; Ryabchikova 2005; Shulyak et al. 2009; Thiam et al. 2010). In addition to these classical approaches to detect abundance stratifications, one has some observations (spectroscopic variabilities) of propagating waves in roAp stars, which unveil high altitude clouds of heavy elements (Mkrtichian et al. 2008). Clouds are also observed through rotational modulation in some spectral lines (see, for instance, Adelman et al. 2002; Ryabchikova et al. 2002; Freyhammer et al. 2009; Briquet et al. 2010). Because such abundance stratifications or clouds cannot exist in presence of mixing, one can say now that stable atmospheres exist.

Among ApBp stars, only roAp stars are well known to pulsate. The situation is less clear for $\mathrm{HgMn}$ stars (in the $T_{\text {eff }}$ range $10000-16000 \mathrm{~K}$ ), which may be considered as a continuation of the AmFm family in the upper main sequence. According to the instability strip for SPB type pulsators calculated by Miglio et al. (2007), HgMn stars may be inside it. However, their calculations are based on homogeneous distribution of metal abundances, which is not the case for HgMn stars. Models calculated by Turcotte \& Richard (2003), with partially stratified abundances, give the same result: HgMn 


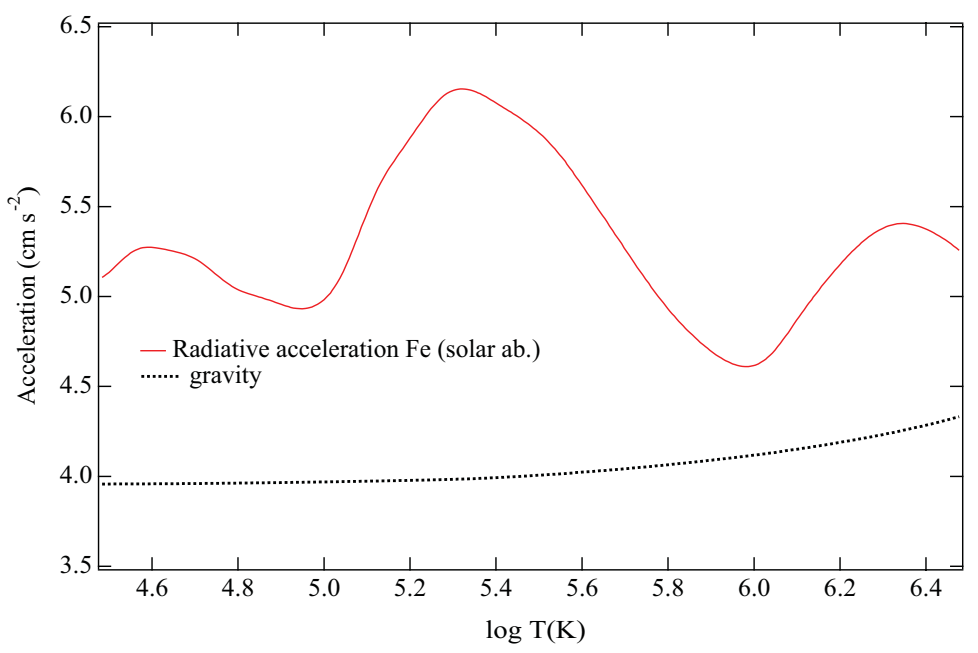

Figure 2. Radiative acceleration on $\mathrm{Fe}$ in a $10 M_{\odot}$ star. The solid (red) line is the logarithm of the radiative acceleration (in $\mathrm{cm} \mathrm{s}^{-2}$ ) plotted vs. the logarithm of the temperature (in $\mathrm{K}$ ). The dotted line is the local gravity.

stars could be SPB-type pulsators, but these evolution models need to be improved. Observations with CoRoT of some HgMn stars show that at least some of them present mono-periodic photometric variations (Alecian et al. 2009; Morel et al. 2013); however, it is not yet established whether these variations are pulsations or rotational modulation.

\subsection{About $\beta$ Cep stars}

There are no chemically peculiar stars hotter than $T_{\text {eff }} \approx 18000 \mathrm{~K}$ (except stars with helium abundance anomalies), because mass-loss rates become too large. However, even if one cannot identify superficial strong abnormal abundances, one cannot exclude that atomic diffusion may affect abundances inside radiative zones of hot stars. To illustrate this possibility, we have computed the radiative acceleration of Fe in a $10 M_{\odot}$ star (Fig. 2) and the corresponding diffusion velocity (Fig. 3). These computations have been carried out using the same code as the one used by Alecian et al. (2013) for Sc, and a model (for an age of about 10 million years, with solar abundances) obtained with the stellar evolution code CLES (Scuflaire 2005). Figure 2 shows that the radiative acceleration is 100 times larger than gravity for layers with $\log T \approx 5.3$ (position of the iron bump). This means that Fe is strongly supported by the radiation field, and so the diffusion velocity is relatively large in these layers (we have neglected here the effect of the thermal diffusion, which will moderate the efficiency of the radiative acceleration). The radiative acceleration on iron at solar abundance (as shown in Fig. 2) is strong enough to support large overabundances of $\mathrm{Fe}$ (about $10^{4}$ times the solar value, if one does a simple extrapolation!). Of course, such huge overabundance will never be reached, first because the diffusion time is too long compared with the evolution time of the star, and second because atomic diffusion competes with other processes. In Fig. 3 the diffusion velocity is compared to a mass-loss velocity computed for this model using Eq. 2.3, for a mass-loss rate of $10^{-12} M_{\odot} \mathrm{yr}^{-1}$. Indeed, the diffusion velocity is large enough to change significantly the Fe abundance in less than $10^{5}$ years $\dagger$. According to these calculations, we see that iron can accumulate inside the radiative zone of a $10 M_{\odot}$ star, provided that the

$\dagger$ The diffusion timescale is defined as the time needed for atoms of the species under consideration to diffuse through a distance of one pressure scale height. 


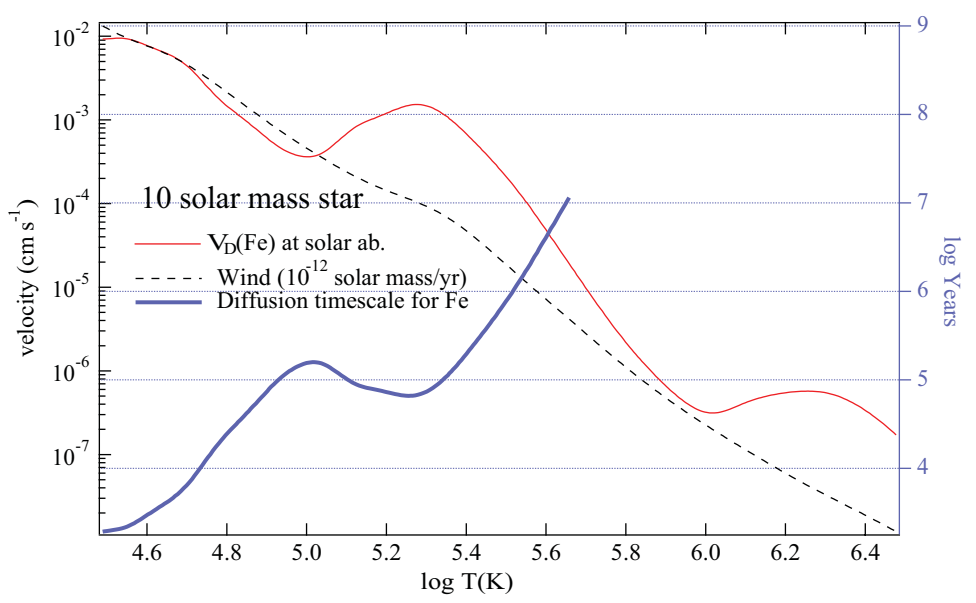

Figure 3. Diffusion velocity, diffusion timescale of Fe, and mass-loss velocity in a $10 M_{\odot}$ star. The diffusion velocity (red solid line) is obtained using the radiative acceleration shown in Fig. 2, the mass-loss velocity (dashed line) corresponds to a mass-loss rate of $10^{-12} M_{\odot} \mathrm{yr}^{-1}$. Left axis corresponds to the velocity (in $\mathrm{cm} \mathrm{s}^{-1}$ ); right axis is the diffusion timescale (blue heavy solid line). The timescale curve ends at about $10^{7}$ years, which is the age of the star for this model.

mass-loss rate is not much larger than $10^{-12} M_{\odot} \mathrm{yr}^{-1}\left(10^{-11} M_{\odot} \mathrm{yr}^{-1}\right.$ seems still acceptable), and that the velocity due to large scale circulations (due to rotation for instance) is not significantly larger than $10^{-3} \mathrm{~cm} \mathrm{~s}^{-1}$ in layers with $\log T \approx 5.3$. A detailed estimate of iron accumulation would require detailed calculation with an evolution code.

Such results could apply to the case of some $\beta$ Cep stars, for which models need larger opacities in the iron bump layers to account for the observed pulsation pattern (Bourge et al. 2006). This is especially interesting for $\beta$ Cep stars with low metallicity because the final local overabundances built up by atomic diffusion in iron-bump layers do not depend on the initial metallicity (except through the internal structure and details of the evolution history of a low-metallicity star). In stars with low metallicity, the diffusion velocities could even be larger, and diffusion timescale shorter than in stars with solar metallicity.

\section{Conclusion}

Because atomic diffusion is a very slow process, and because the abundance inhomogeneities are fragile when faced with the mixing processes usually considered in stellar modeling, the efficiency of atomic diffusion has been often considered with caution, and too often neglected.

Nowadays, it is possible to say that we have a coherent picture of chemically peculiar stars in the framework of atomic diffusion, even if the modeling of individual stars remains a challenge. The important requirement to make this process efficient (the absence of mixing processes) is supported by several observations.

There is no reason to consider that atomic diffusion has visible effects only in chemically peculiar stars. Chemically peculiar stars should be considered as extreme cases, and mild abundance stratifications should exist in all outer radiative zones at least for main-sequence stars heavier than about $1.5 M_{\odot}$ (and at least for layers with $\log T \lesssim 6$. where partial ionization is large enough, and diffusion timescales short enough). By mild abundance stratifications, we mean variations of local abundances by a factor around 2 or 3 for instance. Such a departure from homogeneous abundances, for a given metallicity, 
may have significant effects on models when accurate opacities are required. There are large efforts to make available accurate opacity tables, and a $10 \%$ change in the opacity of iron is considered significant. So, what if the uncertainties in the local abundances are around a factor of two at the iron-bump position? Asteroseismology is certainly a fantastic tool to probe such local properties of stellar interiors, and the case of $\beta$ Cep stars with low metallicity is a perfect illustration of what could be addressed by refining the physics implemented in models.

\section{References}

Adelman, S. J., Gulliver, A. F., Kochukhov, O. P., \& Ryabchikova, T. A. 2002, ApJ, 575, 449

Alecian, G. 1996, A\&BA, 310, 872

Alecian, G. \& Michaud, G. 2005, A\&SA, 431, 1

Alecian, G., Gebran, M., Auvergne, M., et al. 2009, A\& $A$, 506, 69

Alecian, G., LeBlanc, F., \& Massacrier, G. 2013, A\& $A$, 554, A89

Aller, L. H. \& Chapman, S. 1960, ApJ, 132, 461

Bourge, P., Alecian, G., Thoul, A., Scuflaire, R., \& Théado, S. 2006, CoAst, 147, 105

Briquet, M., Korhonen, H., González, J. F., Hubrig, S., \& Hackman, T. 2010, A\&A A, 511, A71

Chapman, S. \& Cowling, T. G. 1970, The Mathematical Theory of non-uniform Gases (3rd ed.; Cambridge: Cambridge University Press)

Charbonneau, P. \& Michaud, G. 1988, ApJ, 327, 809

Freyhammer, L. M., Kurtz, D. W., Elkin, V. G., et al. 2009, MNRAS, 396, 325

Landstreet, J. D. 2011, A\& $A, 528$, A132

Leblanc, F. \& Alecian, G. 2008, A\& A, 477, 243

Michaud, G. 1970, ApJ, 160, 641

Michaud, G., Richer, J., \& Vick, M. 2011, A\&A, 534, A18

Miglio, A., Montalbán, J., \& Dupret, M.-A. 2007, MNRAS, 375, L21

Mkrtichian, D. E., Hatzes, A. P., Saio, H., \& Shobbrook, R. R. 2008, A\&A, 490, 1109

Morel, T., Briquet, M., Auvergne, M., et al. 2013, A\&A, in press

Richer, J., Michaud, G., \& Turcotte, S. 2000, ApJ, 529, 338

Ryabchikova, T. 2005, in: G. Alecian, O. Richard, \& S. Vauclair (eds.), EAS Publications Series, Vol. 17, p. 253

Ryabchikova, T., Piskunov, N., Kochukhov, O., et al. 2002, AछA, 384, 545

Schatzman, E. 1969, A\&SA, 3, 331

Scuflaire, R. 2005, Institut d'Astrophysique et de Geophysique, Université de Liège, Belgium, CLES, Tech. rep.

Shulyak, D., Ryabchikova, T., Mashonkina, L., \& Kochukhov, O. 2009, A\&A, 499, 879

Thiam, M., Leblanc, F., Khalack, V., \& Wade, G. A. 2010, MNRAS, 405, 1384

Turcotte, S. \& Richard, O. 2003, ApESSS, 284, 225

Vick, M., Michaud, G., Richer, J., \& Richard, O. 2010, A\&\&A, 521, A62 